\title{
Bacteriological Profile and Antibiogram of Neonatal Septicemia in a Tertiary Care Hospital
}

\author{
Bipin Gupta ${ }^{1}$, Sneha Mohan ${ }^{1 *}$, Anjali Agarwal ${ }^{2}$ and Renu Dutta ${ }^{1}$ \\ ${ }^{1}$ Department of Microbiology, School of Medical Sciences and Research, Sharda University, \\ Greater Noida, Uttar Pradesh, India \\ ${ }^{2}$ Department of Microbiology, Hind Institute of Medical Sciences, Barabanki, \\ Uttar Pradesh, India \\ *Corresponding author
}

\begin{tabular}{|c|}
\hline Keywords \\
\hline $\begin{array}{l}\text { Neonatal sepsis, } \\
\text { Blood culture, } \\
\text { Antibiogram, } \\
\text { CoNS, Klebsiella }\end{array}$ \\
\hline Article Info \\
\hline $\begin{array}{l}\text { Accepted: } \\
\text { 22 July } 2018 \\
\text { Available Online: } \\
\text { 10 August } 2018\end{array}$ \\
\hline
\end{tabular}

\section{A B S T R A C T}

Septicemia in neonates refers to generalized bacterial infection documented by positive blood culture in the first four weeks of life. Neonatal septicaemia remains one of the most important causes of mortality despite considerable progress in hygiene, introduction of new antimicrobial agents and advanced measures for early diagnosis and treatment. In this cross-sectional study, blood samples from the suspected infants were collected and processed in the bacteriology laboratory. The growth was identified by standard microbiological protocol and the antibiotic sensitivity testing was carried out on MHA by Kirby-Bauer disk diffusion method as recommended in CLSI guidelines. Out of the 147 neonates (M: F = 1.3: 1) admitted to the NICU, 52 (35.4\%) shows blood culture positive. Gram positive was the major organism isolated $46(88.5 \%)$, followed by Gram negative organism $6(11.5 \%)$. CoNS $(63 \%)$ was the predominant Gram positive organism and Klebsiella species $(66.6 \%)$ was the predominant Gram negative organism. Best overall sensitivity among Gram positive isolates was to vancomycin (100\%) and linezolid (100\%). High level resistance was seen against penicillin and fluoroquinolones. Gram negative isolates demonstrated highest sensitivity against imipenem $(100 \%)$ and ciprofloxacin $(100 \%)$. High level resistance was seen against cephalosporins. Neonatal septicaemia is associated with the significant mortality and morbidity. Due to changing micrbiological and antibiotic pattern, a regular surveillance is necessary and blood culture is the gold standard method for diagnosis and should be done in all the suspected cases of neonatal sepsis.

\section{Introduction}

Neonatal sepsis is a clinical syndrome characterized by signs and symptoms of infection with or without accompanying bacteraemia in the first month of life. When pathogenic bacteria gain access into the bloodstream, they may cause overwhelming infection without much localization (septicemia) or may be predominantly localized to the lung (pneumonia) or the meninges (meningitis). Septicemia in neonates refers to generalized bacterial infection documented by positive blood culture in the first four weeks of life (Agnihotri et al., 2004).

Neonatal septicaemia remains one of the most important causes of mortality despite 
considerable progress in hygiene, introduction of new antimicrobial agents and advanced measures for early diagnosis and treatment. (Gotoff, 1996; Haque, 1988) The incidence of neonatal sepsis according to the data from National Neonatal Perinatal Database (NNPD, 2002-03) is 30 per 1000 live births. The NNPD network comprising of 18 tertiary care neonatal units across India found sepsis to be one of the commonest causes of neonatal mortality contributing to $19 \%$ of all neonatal deaths. (http://www.newbornwhocc.org/pdf/ nnpd_report_2002-03.pdf)

Neonatal sepsis is classified as early onset when it occurs within the first 72 hours of life and late onset when it occurs after 72 hours of life (Al-Zwani, 2002; Chacko and Sohi, 2005). Early onset sepsis is caused by organisms prevalent in the maternal genital tract, labour room or operating theatre (Bellig and Ohning, 2013; Zaidi et al., 2008) while late onset sepsis usually results from nosocomial or community-acquired infection (Zaidi et al., 2008; Sankar et al., 2008). Among intramural births, Klebsiella pneumoniae is the most frequently isolated pathogen (32.5\%), followed by Staphylococcus aureus (13.6\%). Among extramural neonates (referred from community/other hospitals), Klebsiella pneumoniae is again the commonest organism (27\%), followed by Staphylococcus aureus (15\%) and Pseudomonas (13\%) (http://www. newbornwhocc.org/pdf/nnpd_report_200203.pdf).

Sepsis is one of the most common causes of neonatal hospital admissions. (Sankar et al., 2008; Darmstadt et al., 2009; Sundaram et al., 2009) Newborns are particularly susceptible to sepsis as a result of their immature immune system, the decreased phagocytic activity of their white blood cells and their incompletely developed skin barriers (Levy, 2007; Shah et al., 2006; Trotman et al., 2006). Common risk factors for neonatal sepsis in Northern India have been identified as low birth weight, perinatal asphyxia, preterm labour and premature rupture of membranes (Roy et al., 2002).

Neonatal sepsis is a medical emergency which presents with subtle, diverse and nonspecific symptoms and signs. Delay in diagnosis and commencement of appropriate treatment may result in high morbidity and mortality rates (Ahmed et al., 2005). Blood culture, which is the gold standard for the diagnosis of sepsis, takes at least 48 hours to obtain preliminary results (Buttery, 2002). It is therefore necessary to initiate an empirical choice of antibiotics based on the epidemiology of causative agents and antibiotic sensitivity patterns in a locality (Asuquo, 1996). Periodic bacterial surveillance is a necessity in every unit because the organisms responsible for neonatal sepsis have been shown to vary across geographical boundaries and with time of onset of illness (Al-Zwani, 2002). So, the present study has been undertaken to determine the bacteriological profile and antimicrobial sensitivity patterns from blood cultures of neonates in our hospital.

\section{Materials and Methods}

The present study was conducted in Microbiology department at tertiary care centre sharda hospital, Greater Noida over a period of one year on 147 neonates admitted in neonatal intensive care unit with clinically suspected septicemia.

Blood sample was collected from a peripheral vein under aseptic conditions. Approximately, 1-3 $\mathrm{ml}$ of blood was inoculated into "BacT/ALERT PF Plus" aerobic pediatric culture bottle aseptically. Blood culture was performed using a Bectec Dickson ped plus aerobic bottles and incubation was performed in Bactec 9240 system. All the bottles were subjected to gram stain and subculture on 
Blood agar and MacConkey Agar. The plates were incubated at $37 \mathrm{oC}$ for $24 \mathrm{hrs}$. Growth was identified by colony morphology, gram stain and standard biochemical tests. (Mackie and McCartney, 2006)

Antimicrobial susceptibility testing was performed on Muller-Hinton agar by KirbyBauer disc diffusion method as recommended in the CLSI guidelines 2014 (CLSI, 2014). Antibiotic disks were procured from Himedia and were penicillin (10 units), cefoxitin (30 $\mu \mathrm{g})$, vancomycin $(30 \mu \mathrm{g})$, amikacin (30 $\mu \mathrm{g})$, erythromycin $(15 \mu \mathrm{g})$, ciprofloxacin (5 $\mu \mathrm{g})$, clindamycin $(2 \mu \mathrm{g})$, linezolid $(30 \mu \mathrm{g})$, amoxicillin/clavulanic acid (20/10 $\mu \mathrm{g})$, cefixime $(5 \mu \mathrm{g})$, cefotaxime $(30 \mu \mathrm{g})$, imipenem $(10 \mu \mathrm{g})$, meropenem $(10 \mu \mathrm{g})$, amikacin $(30 \mu \mathrm{g})$, gentamicin $(10 \mu \mathrm{g})$, ciprofloxacin $(5 \mu \mathrm{g})$ and levofloxacin $(5 \mu \mathrm{g})$.

\section{Results and Discussion}

During the study period, 147 non repeat blood samples were collected from suspected neonatal septicemia patients. Blood culture positive were seen in 52(35.4\%) neonates. Of which $30(57.6 \%)$ cases were male and $22(42.3 \%)$ were female with male to female ratio 1.3:1. Gram positive isolates constituted major group 46(88.5\%) followed by Gram negative isolates 6(11.5\%). Among gram positive isolates, Coagulase Negative Staphylococcus species (CoNS) was found to be the predominant pathogen 29(63\%) followed by Staphylococcus aureus $16(34.7 \%)$. While, among gram negative isolates, Klebsiella species $4(66.6 \%)$ was predominant organism (Table 1).

Antibiogram of gram positive organisms is shown in Table $2.100 \%$ sensitivity was seen against vancomycin and linezolid. Antibiogram of gram negative isolates is shown in Table $3.100 \%$ sensitivity was seen against carbapenems and ciprofloxacin. The changing microbiological patterns of neonatal septicemia warrant the need of monitoring of causative organism and their antibiotic sensitivity pattern. Also the clinical signs and symptoms of neonatal sepsis are subtle and nonspecific, making its early diagnosis difficult. So for effectual management of septicemia cases, study of bacteriological profile along with the antimicrobial sensitivity pattern plays an important role (English et al., 2014; The Young Infant Clinical Study Group, 2008).

In our study, out of 147 clinically suspected cases of sepsis, 52 were culture positive with blood culture positivity rate of $35.4 \%$. There has been a wide variation in growth positivity obtained by blood culture over the years. A high isolation rate was reported by Murty et al., (52.6\%), Roy et al., (47.5\%) and Thakur et al., (47\%). (Murty and Gyaneshwari, 2007; Roy et al., 2002; Thakur et al., 2016) A lower positivity rate $26.6 \%$, was observed by Vrishali muley et al., which was comparable with the present study (Muley et al., 2015). Relative low isolation rate seen in our study may be due to several reasons like administration of antibiotic before blood collection. Even negative blood culture does not exclude sepsis as about $26 \%$ of all neonatal sepsis could be due to anaerobes (Jyothi et al., 2013).

The pathogens most often implicated in neonatal sepsis in developing countries from those seen in developed countries. In our study, the isolation rate of Gram positive and Gram negative organism was $88.5 \%$ and $11.5 \%$ respectively. Similarly, the higher isolation of Gram positive organism has been reported by previous studies (Ballot et al., 2012; Kaufman and Fairchild, 2004; Van den Hoogen et al., 2010; Galhotra et al., 2015). While other authors reported gram negative organism as a predominant organism (Muley et al., 2015; Jyothi et al., 2013). The 
predominance of gram positive organism in about infection control measure among our study may be due to many reasons like Healthcare providers (Thakur et al., 2016). overcrowding in NICU, lack of knowledge

Table.1 Species distribution

\begin{tabular}{|l|}
\hline Organisms \\
\hline Gram Positive Cocci \\
\hline CoNS \\
S. aureus \\
Enterococcus \\
\hline Gram Negative Bacilli \\
\hline Klebsiella species \\
E. coli \\
\hline Total
\end{tabular}

Number $(\%)$
$\mathbf{4 6}(\mathbf{8 8 . 5})$
$29(63)$
$16(34.7)$
$1(2.1)$
$\mathbf{6}(\mathbf{1 1 . 5 )}$
$4(66.6)$
$2(33.3)$
$\mathbf{5 2}(\mathbf{1 0 0 \%})$

Table.2 Antibiotic resistant profile of Gram positive organisms

\begin{tabular}{|c|c|c|c|}
\hline \multirow[t]{2}{*}{ Antibiotics } & \multicolumn{3}{|c|}{ Organism } \\
\hline & $\operatorname{CoNS}(\%)(\mathrm{n}=29)$ & S. aureus $(\%)(\mathrm{n}=16)$ & Enterococcus $(\%)(\mathrm{n}=1)$ \\
\hline Penicillin & 82.7 & 87.5 & 100 \\
\hline Cefoxitin & 55.1 & 37.5 & - \\
\hline Vancomycin & - & - & - \\
\hline Amikacin & 6.8 & 6.2 & 100 \\
\hline Erythromycin & 55.1 & 56.2 & 100 \\
\hline Ciprofloxacin & 37.9 & 37.5 & - \\
\hline Clindamycin & 31 & 56.2 & 100 \\
\hline Linezolid & - & - & - \\
\hline
\end{tabular}

Table.3 Antibiotic resistance pattern of Gram negative bacilli (GNB)

\begin{tabular}{|l|}
\multicolumn{1}{|c|}{ Antibiotics } \\
\hline Amoxycillin/Clavulanic acid \\
\hline Cefixime \\
\hline Cefotaxime \\
\hline Imipenem \\
\hline Meropenem \\
\hline Amikacin \\
\hline Gentamicin \\
\hline Ciprofloxacin \\
\hline Levofloxacin \\
\hline
\end{tabular}

\begin{tabular}{|c|c|}
\hline \multicolumn{2}{|c|}{ Organism } \\
\hline Klebsiella $(\%)(n=4)$ & E. coli $(\%)(n=2)$ \\
\hline 75 & $50 \%$ \\
\hline 100 & 50 \\
\hline 75 & 100 \\
\hline - & - \\
\hline - & - \\
\hline- & 50 \\
\hline 50 & 50 \\
\hline - & - \\
\hline 25 & 50 \\
\hline
\end{tabular}


CoNS (63\%) was the predominant gram positive organism isolated in this study. Similarly, Gheibi et al., reported CoNS $(54.6 \%)$ as predominant gram positive organism (Gheibi et al., 2008). Some of the previous studies also observed CoNS as their predominant pathogen (Muley et al., 2015; Sneha Ann Oommen et al., 2015) In present study, S.aureus was isolated from $34.7 \%$ cases and was the next common pathogen following CoNS. While $S$. aureus was reported as predominant pathogen by (Thakur et al., 2016) most common gram negative organism isolated in our study was Klebseilla species $(66.6 \%)$, which was comparable with the previous studies findings (Roy et al., 2002; Muley et al., 2015).

This change of bacteriological profile from predominant gram negative to predominant gram positive isolation has been observed worldwide. Many recent studies have reported the emergence of new emerging organism such as CoNS, Candida species as a cause of neonatal sepsis (Thakur et al., 2016). The colonization of skin and nasopharynx by CoNS and S.aureus in healthcare workers and improper hand washing technique leading to horizontal transmission to neonates further leads to increase in isolation rate of gram positive organism in them (Thakur et al., 2016)

In our study, a male preponderance was seen with male to female ratio of 1.3:1 which was in concordance with previous studies (Jyothi et al., 2013; Galhotra et al., 2015). This might be because of more number of male infants born compared to female infants born.

The antibiotic sensitivity pattern differs in different studies at different times in the same hospital worldwide. This is mainly due to indiscriminate use of antibiotics (Tsering et al., 2011). In present study, maximum number of organism was resistant to commonly used antibiotics. Antibiogram of our study revealed that majority of Gram positive isolates (CoNS and S. aureus) were resistant to penicillin (82.7\% and $87.5 \%)$. Similar findings were reported by (Roy et al., 2002; Jyothi et al., 2013; Tsering et al., 2011) All gram positive isolates showed $100 \%$ sensitivity against Vancomycin and Linezolid, which was in concordance with the previous studies findings (Roy et al., 2002; Jyothi et al., 2013). Hence, these drugs can be effectively be used in multi-drug resistance cases.

Among gram negative isolates, Klebsiella spps and E.coli resistant pattern were as follows respectively; Amoxycillin/clavulinic acid $(75 \%$ and $50 \%)$, Cefotaxime (75\% and $100 \%)$, Cefixime (100\% and 50\%), Gentamicin (50\% and 50\%), Levofloxacin (25\% and 50\%). All gram negative isolates were $100 \%$ sensitive to carbapenems. Gram negative isolates showed resistance to $\beta$ lactam combination antibiotics and extended spectrum cephalosporins at high level. Similarly, high level resistance was reported by Roy et al., (2002), Jyothi et al., (2013), Galhotra et al., (2015). Therefore, these drugs can't be used as empiric treatment for neonatal sepsis. However, low resistance was seen against flouroquinolones and carbapenems. These drugs can be used as empirical therapy in order to prevent multidrug resistance, but it should be used cautiously.

The microbiological pattern of neonatal septicemia is a changing landscape and is associated with significant morbidity and mortality, including long term morbidity. Therefore, there is need of regular periodic surveillance of the causative organisms of neonatal sepsis as well as their antibiotic susceptibility patterns to inform the choice of empirical antibiotic treatment while awaiting blood culture results. CoNS and Klebsiella 
was observed to be the leading cause of neonatal sepsis in our study and were resistance to commonly used antibiotics. Therefore, regular monitoring of antibiotic resistance is necessary and depending on the antibiotic sensitivity pattern of the isolates, antibiotic should be used. Blood culture is a Gold Standard for diagnosis of neonatal sepsis and should be done in all the suspected cases of neonatal sepsis.

Furthermore, health education should be provided to the public on the dangers of indiscriminate use of antibiotics, which is currently considered to be a menace in our society and which has been responsible for the ineffectiveness of most commonly used antibiotics such as penicillin, as observed in our study.

\section{References}

Agnihotri N, Kaistha N, Gupta V. Antimicrobial susceptibility of isolates from neonatal septicemia. Jpn J Infect Dis 2004; 57: 273-75.

Ahmed Z, Ghafoor T, Waqar $\mathrm{T}$, et al., Diagnostic value of C-reactive protein and hematological parameters in neonatal sepsis. J Coll Phys Surg Pak 2005; 15(3): 152-56.

Al-Zwani EJK. Neonatal septicaemia in the neonatal care unit, Al-Anbar Governorate, Iraq. East Medit Health J 2002; 8: 4-5.

Asuquo UA. Antibiotic therapy in neonatal septicaemia (Editorial).Niger JPaediatr 1996; 23: 1-3.

Ballot DE, Nana T, Sriruttan C, Cooper PA. Bacterial bloodstream infections in neonates in a developing country. ISRN Pediatr 2012; 2012: 508512.

Bellig LL, and Ohning BL. Neonatal Sepsis. [Retrieved $6^{\text {th }}$ June 2013]. http://www. emedicine.com/ped/topic2630.html.

Buttery JP. Blood cultures in newborns and children: optimizing an everyday test. Arch Dis Child Fetal Neonatal Ed 2002; 87(1): 25-28.
Chacko B, and Sohi I. Early onset sepsis. Indian J Pediatr 2005; 72(1): 23-26.

Clinical and Laboratory Standards Institute. Performance Standards for Antimicrobial Susceptibility Testing; Twenty-Third Informational Supplement. CLSI document M100-S23. Wayne, PA: Clinical and Laboratory Standards Institute 2014.

Darmstadt GL, Batra M, Zaida AKM. Parenteral antibiotics for the treatment of serious neonatal bacterial infections in developing country settings. Pediatr Infect Dis J 2009; 28(1):37-42.

English, M., M. Ngama, L. Mwalekwa, N. Peshu. Signs of illness in Kenyan infants aged less than 60 days. Bulletin of the World Health Organization 2004; 82(5): 323-29.

Galhotra S, Gupta V, Bains HS, Chhina D. Clinico-bacteriological profile of neonatal septicemia in tertiary care hospital. J Mahatma Gandhi Inst Med Sci 2015; 20: 148-52.

Gheibi S, Fakoor Z, Karamyyar M, Khashabi J, Ilkhanizadeh B, Sana AF, et al., Coagulase negative staphylococcus; the most common cause of neonatal septicaemia in Urmia, Iran. Iran J Pediatr 2008; 18: 237-43.

Gotoff SP. Neonatal sepsis and meningitis: In: Nelson Textbook of Pediatrics (15th Edition). Eds Behrman RE, Kleigman RM, Arvin AM. Philadelphia, WB Saunders Company, 1996; 528-37.

Haque KH. Infection and immunity in the newborn. In: Forfar and Arneil's Textbook of Pediatrics ( $5^{\text {th }}$ Edition). Eds Campbell AGM, Macintosh N. Pearson Professional Limited, 1988; 273-89.

Jyothi, P., Metri C. Basavaraj and Peerapur V. Basavaraj, Bacteriological profile of neonatal septicemia and antibiotic susceptibility pattern of the isolates, Journal of Natural Science Biology and Medicine 2013 Vol-4 (306-309).

Kaufman D, and Fairchild KD. Clinical microbiology of bacterial and fungal 
sepsis in very-low-birth-weight infants. Clin Microbiol Rev 2004; 17: 638-80.

Levy O. Innate immunity of the newborn: basic mechanisms and clinical correlates. Nat Rev Immunol 2007; 7(5): 379-90.

Mackie and McCartney Practical Medical Microbiology, Tests for the identification of Bacteria, $14^{\text {th }}$ Edition, Delhi: Elsevier Publication 2006, 131-50.

Muley VA, Ghadage DP, Bhore AV. Bacteriological profile of neonatal septicemia in a tertiary care hospital from Western India. J Global Infect Dis 2015; 7: 75-7

Murty DS, and Gyaneshwari M. Blood cultures in pediatric patients: A study of clinical impact. Indian J Med Microbiol. 2007; 25: $220-4$

National Neonatal Perinatal Database. Report for the year 2002-03. http://www.newbornwhocc.org/pdf/nnpd_ report_2002-03.pdf.

Roy I, Jain A, Kumar M, Agrawal SK. Bacteriology of neonatal septicemia in a tertiary care hospital of northern India. Indian J Med Microbiol 2002; 20: 156-9.

Sankar MJ, Agarwal R, Deorari AK, et al., Sepsis in the newborn. Indian J Pediatr 2008; 75(3): 261-72.

Shah GS, Budhathoki S, Das BK, et al., Risk factors in early neonatal sepsis. Kathmandu Univ Med J 2006; 4(2): $187-$ 91.

Sneha Ann Oommen, Santosh Saini, Kunkulol Rahul R. Bacteriological profile of neonatal septicemia: a retrospective analysis from a tertiary care hospital in loni. Int J Med Res Health Sci. 2015; 4(3):652-658.

Sundaram V, Kumar P, Dutta S, et al., Blood culture confirmed bacterial sepsis in neonates in North Indian tertiary care centre: changes over the last decade. Jpn J Infect Dis 2009; 62(1): 46-50.

Thakur S, Thakur K, Sood A, Chaudhary S. Bacteriological profile and antibiotic sensitivity pattern of neonatal septicemia in a rural tertiary care hospital in North India. Indian J Med Microbiol 2016; 34: 6-71.

The Young Infant Clinical Study Group. Clinical signs that predict severe illness in children under age 2 months: a multicenter study. The Lancet 2008; 371(9607): 135-42.

Trotman H, Bell Y, Thame M, et al., Predictor of poor outcome in neonates with bacterial sepsis admitted to the University Hospital of the West Indies. West Indian Med J 2006; 55(2): 80-83.

Tsering DC, Chanchal L, Pal R, Kar S. Bacteriological profile of septicemia and the risk factors in neonates and infants in Sikkim. J Global Infect Dis 2011; 3: 42-5.

Van den Hoogen A, Gerards LJ, VerboonMaciolek MA, Fleer A, Krediet TG. Long-term trends in the epidemiology of neonatal sepsis and antibiotic susceptibility of causative agents. Neonatology 2010; 97: 22-8.

Zaidi AK, Thaver D, Ali SA, et al., Pathogens associated with sepsis in newborns and young infants in developing countries. Pediatr Infect Dis J 2008; 28(1): 10-18.

\section{How to cite this article:}

Bipin Gupta, Sneha Mohan, Anjali Agarwal and Renu Dutta. 2018. Bacteriological Profile and Antibiogram of Neonatal Septicemia in a Tertiary Care Hospital. Int.J.Curr.Microbiol.App.Sci. 7(08): 3999-4005. doi: https://doi.org/10.20546/ijcmas.2018.708.414 